\title{
The capacity associated to signed Riesz kernels, and Wolff potentials.
}

\author{
Joan Mateu, Laura Prat and Joan Verdera.
}

\begin{abstract}
We show that, for $0<\alpha<1$, the capacity associated to the signed vector valued Riesz kernel $\frac{x}{|x|^{1+\alpha}}$ in $\mathbb{R}^{n}$ is comparable to the Riesz capacity $C_{\frac{2}{3}(n-\alpha), \frac{3}{2}}$ of non-linear potential theory.
\end{abstract}

\section{Introduction.}

In this paper we study the capacity $\gamma_{\alpha}$ associated to the signed vector valued Riesz kernels $k_{\alpha}(x)=\frac{x}{|x|^{1+\alpha}}, 0<\alpha<n$, in $\mathbb{R}^{n}$. If $K \subset \mathbb{R}^{n}$ is compact one sets

$$
\gamma_{\alpha}(K)=\sup |<T, 1>|,
$$

where the supremum is taken over all distributions $T$ supported on $K$ such that $T * \frac{x_{i}}{|x|^{1+\alpha}}$ is a function in $L^{\infty}\left(\mathbb{R}^{n}\right)$ and $\left\|T * \frac{x_{i}}{|x|^{1+\alpha}}\right\|_{\infty} \leq 1$, for $1 \leq i \leq n$. For $n=2$ and $\alpha=1$ this is basically analytic capacity (see [T1]), and for $\alpha=n-1$ and any $n \geq 2, \gamma_{n-1}$ is Lipschitz harmonic capacity (see [Par], [MP] and [V1]).

In $[\mathrm{P}$ one discovered the fact that if $0<\alpha<1$, then a compact set of finite $\alpha$-dimensional Hausdorff measure has zero $\gamma_{\alpha}$ capacity. This is in strong contrast with the situation for integer $\alpha$, in which $\alpha$-dimensional smooth hypersurfaces have positive $\gamma_{\alpha}$ capacity. The case of non-integer $\alpha>1$ is not completely understood, although it was shown in $[\mathbb{P}$ that for Ahlfors-David regular sets the result mentioned above for $0<\alpha<1$ still holds.

In this paper we establish the equivalence between $\gamma_{\alpha}, 0<\alpha<1$, and one of the well-known Riesz capacities of non-linear potential theory (see [AH], Chapter 1, p. 38). The Riesz capacity $C_{s, p}$ of a compact set $K \subset \mathbb{R}^{n}, 1<p<\infty, 0<s p \leq n$, is defined by

$$
C_{s, p}(K)=\inf \left\{\|\varphi\|_{p}^{p}: \varphi * \frac{1}{|x|^{n-s}} \geq 1 \text { on } K\right\},
$$

where the infimum is taken over all compactly supported infinitely differentiable functions on $\mathbb{R}^{n}$. The capacity $C_{s, p}$ plays a central role in understanding the nature of Sobolev spaces (see $\underline{\mathrm{AH}}]$ ). 
Our main result is the following surprising inequality.

Theorem. For each compact set $K \subset \mathbb{R}^{n}$ and for $0<\alpha<1$ we have

$$
C^{-1} C_{\frac{2}{3}(n-\alpha), \frac{3}{2}}(K) \leq \gamma_{\alpha}(K) \leq C C_{\frac{2}{3}(n-\alpha), \frac{3}{2}}(K),
$$

where $C$ is a positive constant depending only on $\alpha$ and $n$.

Since it is well-known that $C_{\frac{2}{3}(n-\alpha), \frac{3}{2}}$ vanishes on sets of finite $\alpha$-dimensional Hausdorff measure (see [AH], Theorem 5.1.9, p.134), the same applies to $\gamma_{\alpha}$. Thus we recover one of the main results of $[\mathrm{P}]$. On the other hand, $C_{s, p}$ is a subadditive set function (almost by definition, see $\mathrm{AH}$, p.26), and consequently, $\gamma_{\alpha}$ is semiadditive for $0<\alpha<1$, that is, given compact sets $K_{1}$ and $K_{2}$,

$$
\gamma_{\alpha}\left(K_{1} \cup K_{2}\right) \leq C\left\{\gamma_{\alpha}\left(K_{1}\right)+\gamma_{\alpha}\left(K_{2}\right)\right\},
$$

for some constant $C$ depending only on $\alpha$ and $n$. In fact $\gamma_{\alpha}$ is countably semiadditive. For $\alpha=1$ and $n=2$ inequality (11) is still true and is a remarkable result obtained in [T1]. For $\alpha=n-1$ and any $n$ (11) has been shown very recently in [Vo].

Another interesting consequence of the Theorem is that $\gamma_{\alpha}$ is a bilipschitz invariant. This means that if $\phi: \mathbb{R}^{n} \rightarrow \mathbb{R}^{n}$ is a bilipschitz homeomorphism of $\mathbb{R}^{n}$, namely,

$$
L^{-1}|x-y| \leq|\phi(x)-\phi(y)| \leq L|x-y|,
$$

for $x, y \in \mathbb{R}^{n}$ and for some constant $L>0$, then for compact sets $K$ one has

$$
C^{-1} \gamma_{\alpha}(K) \leq \gamma_{\alpha}(\phi(K)) \leq C \gamma_{\alpha}(K)
$$

where $C$ depends only on $L, \alpha$ and $n$.

The bilipschitz invariance of the analytic capacity $\gamma$ has been recently proved by X. Tolsa (see [T3]). The result for a big class of Cantor sets was proved before by Garnett and Verdera (see GV]).

Volberg has pointed out to the authors that a particular instance of the Theorem gives the following curious result about Cauchy integrals. Take $n=2$ and $\alpha=\frac{1}{2}$. Then, given a compact set $K \subset \mathbb{C}$, there exists a distribution $T \neq 0$ supported on $K$ such that $T * \frac{z}{|z|^{3 / 2}} \in L^{\infty}(\mathbb{C})$ if and only if there exists a probability measure $\mu$ supported on $K$ such that $\mu * \frac{1}{z} \in L 3(\mathbb{C})$. This follows from the dual definition of $C_{1, \frac{3}{2}}$ (see $[\mathrm{AH}$, Theorem 2.2.7.).

Our proof of the Theorem rests on two steps. The first one is the analogue for $0<\alpha<1$ of the main result in [T1, namely, the equivalence between $\gamma_{\alpha}$ and $\gamma_{\alpha,+}$. For a compact set $K \subset \mathbb{R}^{n}$, the positive $\gamma_{\alpha}$ capacity is defined by

$$
\gamma_{\alpha,+}(K)=\sup \mu(K)
$$


where the supremum is taken over those positive Radon measures $\mu$ supported on $K$ such that $\frac{x_{i}}{|x|^{1+\alpha}} * \mu$ is in $L^{\infty}\left(\mathbb{R}^{n}\right)$ and $\left\|\frac{x_{i}}{|x|^{1+\alpha}} * \mu\right\|_{\infty} \leq 1$, for $1 \leq i \leq n$. Clearly $\gamma_{\alpha,+}(K) \leq \gamma_{\alpha}(K)$ for any $K$.

Theorem 1.1. For each compact set $K \subset \mathbb{R}^{n}$ and $0<\alpha<1$, we have

$$
\gamma_{\alpha,+}(K) \leq \gamma_{\alpha}(K) \leq C \gamma_{\alpha,+}(K)
$$

where $C$ is some positive constant depending only on $\alpha$ and $n$.

We claim that Theorem 1.1 can be proved by adapting the scheme of the proof of Theorem 1.1 in [T1] and the adjustments introduced in [T2] to prove Theorem 7.1 there. This is explained in some detail in section 2.2. When analyzing the argument used in [T1] one realizes that it is based on two main technical ingredients. The first is the non-negativity of the quantity obtained when symmetrizing the kernel, which was proved in $[\mathrm{P}]$ for the Riesz kernel $k_{\alpha}$ with $0<\alpha<1$. The second is the fact that the Cauchy kernel ( that is, $k_{1}$ in dimension $n=2$ ) localizes in the uniform norm. By this we mean that if $T$ is a compactly supported distribution such that $T * \frac{1}{z}$ is a bounded function then $(\varphi T) * \frac{1}{z}$ is also bounded for each compactly supported $\mathcal{C}^{1}$ function $\varphi$ and we have the corresponding estimate. This is an old result, which is simple to prove because $\frac{1}{z}$ is related to the differential operator $\bar{\partial}([\mathrm{G}$, Chapter $\mathrm{V})$. The same localization result can be proved easily for any $n$ and $\alpha=n-1$, because $k_{n-1}$ is related to the Laplacian ([$[\mathrm{Par}]$ and $\left.[\mathrm{V} 1]\right)$. For other parameters $\alpha$ between 0 and $n$ is not clear at all that there is a differential operator in the background and consequently the corresponding localization result becomes far from being obvious. In fact, the proof of the localization Theorem for $k_{\alpha}$ for any $\alpha, 0<\alpha<n$, is the main technical obstacle we have to surmount in this paper. When localization is available there is no obstruction in adapting Lemma $7.2($ part $(h))$ in [T2]. Once Theorem 1.1 is at our disposal we need to relate $\gamma_{\alpha,+}$ to $C_{\frac{2}{3}(n-\alpha), \frac{3}{2}}$ and this is the second step in the proof of the Theorem.

The plan of the paper is the following. Section 2 contains some preliminary definitions and results that will be used throughout the article. In section 3 we prove the localization theorem for the signed Riesz potentials. In section 4 we complete the proof of the main Theorem showing that $\gamma_{\alpha,+}$ is comparable to $C_{\frac{2}{3}(n-\alpha), \frac{3}{2}}$.

Constants independent of the relevant parameters are denoted by $C$ and may be different at each occurrence. The notation $A \approx B$ means, as it is usual, that for some constant $C$ one has $C^{-1} B \leq A \leq C B$. 


\section{Preliminaries.}

\subsection{Simmetrization of Riesz kernels.}

The symmetrization process for the Cauchy kernel introduced in $[\mathrm{Me}$ has been successfully applied in these last years to many problems of analytic capacity and $L 2$ boundedness of the Cauchy integral operator (see [MV], [MMV] for example; the survey $[\mathrm{D}]$ and the book $[\mathrm{Pa}]$ contain many other interesting references). Given 3 distinct points in the plane, $z_{1}, z_{2}$ and $z_{3}$, one finds out, by an elementary computation that

$$
c\left(z_{1}, z_{2}, z_{3}\right) 2=\sum_{\sigma} \frac{1}{\left(z_{\sigma(1)}-z_{\sigma(3)}\right) \overline{\left(z_{\sigma(2)}-z_{\sigma(3)}\right)}}
$$

where the sum is taken over the six permutations of the set $\{1,2,3\}$ and $c\left(z_{1}, z_{2}, z_{3}\right)$ is Menger curvature, that is, the inverse of the radius of the circle through $z_{1}, z_{2}$ and $z_{3}$. In particular (2) shows that the sum on the right hand side is a non-negative quantity.

It can be shown that for $0<\alpha<1$ the symmetrization of the Riesz kernel $k_{\alpha}(x)=x /|x|^{1+\alpha}$, gives also a positive quantity. On the other hand, for $1<\alpha<n$, the phenomenon of change of signs appears when symmetrizing the kernel $k_{\alpha}$, as one can easily check.

For $0<\alpha<n$ the quantity

$$
\sum_{\sigma} \frac{x_{\sigma(2)}-x_{\sigma(1)}}{\left|x_{\sigma(2)}-x_{\sigma(1)}\right|^{1+\alpha}} \frac{x_{\sigma(3)}-x_{\sigma(1)}}{\left|x_{\sigma(3)}-x_{\sigma(1)}\right|^{1+\alpha}},
$$

where the sum is taken over the six permutations of the set $\{1,2,3\}$, is the obvious analogue of the right hand side of (2) for the Riesz kernel $k_{\alpha}$. Notice that (3) is exactly

$$
2 p_{\alpha}\left(x_{1}, x_{2}, x_{3}\right),
$$

where $p_{\alpha}\left(x_{1}, x_{2}, x_{3}\right)$ is defined as the sum in (3) taken only on the three permutations $(1,2,3),(2,3,1),(3,1,2)$.

In the following lemma we state the explicit description that was found in $\mathbb{P}$ for the symmetrization of the Riesz kernel $k_{\alpha}$, for $0<\alpha<1$.

Lemma 2.1. Let $0<\alpha<1$, and $x_{1}, x_{2}, x_{3}$ three distinct points in $\mathbb{R}^{n}$. Then we have

$$
\frac{2-2^{\alpha}}{L\left(x_{1}, x_{2}, x_{3}\right)^{2 \alpha}} \leq p_{\alpha}\left(x_{1}, x_{2}, x_{3}\right) \leq \frac{2^{1+\alpha}}{L\left(x_{1}, x_{2}, x_{3}\right)^{2 \alpha}},
$$

where $L\left(x_{1}, x_{2}, x_{3}\right)$ is the largest side of the triangle determined by $x_{1}, x_{2}$ and $x_{3}$. In particular $p_{\alpha}\left(x_{1}, x_{2}, x_{3}\right)$ is a positive quantity. 
The relationship between the quantity $p_{\alpha}(x, y, z)$ and the $L 2$ estimates of the operator with kernel $k_{\alpha}$ is as follows. Take a positive finite Radon measure $\mu$ in $\mathbb{R}^{n}$ which satisfies the growth condition $\mu(B(x, r)) \leq r^{\alpha}, x \in \mathbb{R}^{n}, r>0$. Given $\varepsilon>0$, set

$$
R_{\alpha, \varepsilon}(\mu)(x)=\int_{|y-x|>\varepsilon} k_{\alpha}(y-x) d \mu(y) .
$$

Then (see in [MV] or $[\mathrm{Pa}$ the argument for $\alpha=1$ )

$$
\left.\left|\int\right| R_{\alpha, \varepsilon}(\mu)(x)\right|^{2} d \mu(x)-\frac{1}{3} p_{\alpha, \varepsilon}(\mu) \mid \leq C\|\mu\|,
$$

where $C$ is a constant depending only on $\alpha$ and $n$, and

$$
p_{\alpha, \varepsilon}(\mu)=\iiint_{S_{\varepsilon}} p_{\alpha}(x, y, z) d \mu(x) d \mu(y) d \mu(z),
$$

with

$$
S_{\varepsilon}=\{(x, y, z):|x-y|>\varepsilon,|x-z|>\varepsilon \text { and }|y-z|>\varepsilon\} .
$$

Thus

$$
p_{\alpha}(\mu) \leq 3 \sup _{\varepsilon>0} \int\left|R_{\alpha, \varepsilon}(\mu)(x)\right|^{2} d \mu(x)+C\|\mu\|
$$

where

$$
p_{\alpha}(\mu)=\int_{\mathbb{R}^{n}} \int_{\mathbb{R}^{n}} \int_{\mathbb{R}^{n}} p_{\alpha}(x, y, z) d \mu(x) d \mu(y) d \mu(z) .
$$

\subsection{The scheme of the proof of Theorem 1.1.}

In this section we give an outline of the arguments involved in the proof of Theorem 1.1. The proof uses an induction argument on scales, analogous to the one in [MTV] and [T1. The main idea is to show, by induction, that

$$
\gamma_{\alpha,+}(K \cap Q) \approx \gamma_{\alpha}(K \cap Q)
$$

for squares $Q$ of any size.

The starting point in the proof of Theorem 1.1 in [T1] is the construction of a special family of cubes $\left\{Q_{j}\right\}_{j=1}^{N}$ that cover $K$ and satisfy

$$
\gamma_{\alpha,+}\left(\cup_{j=1}^{N} Q_{j}\right) \leq C \gamma_{\alpha,+}(K)
$$


and

$$
\sum_{j=1}^{N} \gamma_{\alpha,+}\left(3 Q_{j} \cap K\right) \leq C \gamma_{\alpha,+}(K) .
$$

The construction of these cubes works without difficulty in the same way as in T1 for $0<\alpha<1$, because we have non-negativity of the quantity obtained when symmetrizing the Riesz kernel (see Lemma 2.1 above).

¿From the definition of the capacity $\gamma_{\alpha}$, it follows that there exists a distribution $T_{0}$ supported on $K$ such that
1. $\gamma_{\alpha}(K) \geq \frac{1}{2}\left|\left\langle T_{0}, 1\right\rangle\right|$
2. $\left\|T_{0} * \frac{x_{i}}{|x|^{1+\alpha}}\right\|_{\infty} \leq 1, \quad 1 \leq i \leq n$.

Consider now a family of infinitely differentiable functions $\left\{\varphi_{j}\right\}_{j=1}^{N}$ such that each $\varphi_{j}$ is compactly supported on $2 Q_{j}, 0 \leq \varphi_{j} \leq 1,\left\|\partial^{s} \varphi_{j}\right\|_{\infty} \leq \frac{C}{\ell\left(Q_{j}\right)^{|s|}}, 0 \leq|s| \leq n$, and $\sum_{j=1}^{N} \varphi_{j}=1$ on $\cup_{j=1}^{N} Q_{j}$. At this point we need an inequality of the type

$$
\left\|\varphi_{j} T_{0} * \frac{x_{i}}{|x|^{1+\alpha}}\right\|_{\infty} \leq C
$$

for $1 \leq i \leq n, 1 \leq j \leq N$ and $0<\alpha<n$, with $C=C(\alpha, n)$. This will be proved in section 3. Then, by definition of $\gamma_{\alpha}$, we will obtain that

$$
\left|\left\langle\varphi_{j} T_{0}, 1\right\rangle\right| \leq C \gamma_{\alpha}\left(2 Q_{j} \cap K\right)
$$

for $1 \leq j \leq N$

Inequality (5) is used later on in the proof in order to construct a bounded function $b$ to which a suitable variant of the $T(b)$ theorem will be applied. There is still one more difficulty in applying the Nazarov, Treil and Volberg $T(b)$-type theorem one needs, namely, finding a substitute for what they call the suppressed operators. It was already explained in $[\mathrm{P}$ that there are at least two versions of such operators for the Riesz kernels that work appropriately.

\section{Localization of Riesz potentials.}

One of the ingredients of the proof of Theorem 1.1 in T1 is the localization of the Cauchy potential. The localization method for the Cauchy potential, $T * 1 / z$, developed by A.G. Vitushkin for rational approximation was adapted in [Par] to localize the potential $T * x /|x|^{n}$ and used in problems of $\mathcal{C}^{1}$-harmonic approximation. 
In this section we will be concerned with the localization of the vector valued $\alpha$-Riesz potentials $T * x /|x|^{1+\alpha}, 0<\alpha<n$.

Let $x=\left(x_{1}, \ldots, x_{n}\right) \in \mathbb{R}^{n}$ and $|x|=\left(\sum_{i=1}^{n} x_{i} 2\right)^{1 / 2}$. For $s=\left(s_{1}, \ldots, s_{n}\right), \quad 0 \leq$ $s_{i} \in \mathbb{Z}$, we set $x^{s}=x_{1}^{s_{1}} \cdots x_{n}^{s_{n}}, s !=s_{1} ! \cdots s_{n} !,|s|=s_{1}+s_{2}+\cdots+s_{n}$, $\partial^{s}=\partial^{s_{1}} / \partial x_{1}^{s_{1}} \cdots \partial^{s_{n}} / \partial x_{n}^{s_{n}}, \Delta=\sum_{i=1}^{n} \partial 2 / \partial x_{i} 2$ and $\partial_{j}=\partial / \partial x_{j}, 1 \leq j \leq n$. In what follows, given a cube $Q \subset \mathbb{R}^{n}, \varphi_{Q}$ will denote an infinitely differentiable function supported on $2 Q$ and such that $\left\|\partial^{s} \varphi_{Q}\right\|_{\infty} \leq C_{s} \ell(Q)^{-|s|}, 0 \leq|s| \leq n$.

We prove now the following general localization lemma.

Lemma 3.1. Let $0<\alpha<n$ and let $T$ be a compactly supported distribution such that $T * \frac{x_{i}}{|x|^{1+\alpha}}$ is a bounded measurable function for $1 \leq i \leq n$. Then there exists some constant $C=C(n, \alpha)>0$ such that

$$
\sup _{1 \leq i \leq n}\left\|\varphi_{Q} T * \frac{x_{i}}{|x|^{1+\alpha}}\right\|_{\infty} \leq C \sup _{1 \leq i \leq n}\left\|T * \frac{x_{i}}{|x|^{1+\alpha}}\right\|_{\infty}
$$

Proof. Our argument uses a reproduction formula for test functions involving the kernel $k^{i}(y)=\frac{y_{i}}{|y|^{1+\alpha}}$, which was first introduced in $[\mathrm{P}]$ (see Lemma 11). There are many variants of this formula depending, for instance, on whether the dimension $n$ and the integer part of $\alpha$ are even or odd. We will consider in full detail only the case of odd dimension of the form $n=2 k+1$. We will also assume that $\alpha$ is non-integer and that its integer part is even, of the form $[\alpha]=2 d$. At the end of the proof we shall briefly indicate how to treat the remaining cases, including the case of integer $\alpha$.

Fix $x \in \mathbb{R}^{n}$ and set

$$
k_{x}^{i}(y)=\frac{x_{i}-y_{i}}{|x-y|^{1+\alpha}}
$$

We distinguish two cases:

Case 1: $x \in(3 Q)^{c}$. Set $g(y)=\varphi_{Q}(y) k_{x}^{i}(y)$. Lemma 11 in $[\mathrm{P}$ tells us that

$$
g(x)=c_{n, \alpha} \sum_{j=1}^{n}\left(\Delta^{k} \partial_{j} g * \frac{1}{|y|^{n-\alpha}} * k^{j}\right)(x),
$$

for some constant $c_{n, \alpha}$ depending only on $n$ and $\alpha$. We emphasize that (6) works because $n$ is odd. Thus

$$
\left(\varphi_{Q} T * k^{i}\right)(x)=<T, g>=c_{n, \alpha} \sum_{j=1}^{n}<T * k^{j}, \Delta^{k} \partial_{j} g * \frac{1}{|y|^{n-\alpha}}>
$$


and so

$$
\begin{aligned}
& \left(\varphi_{Q} T * k^{i}\right)(x)=\sum_{j=1}^{n} c_{n, \alpha} \int_{(3 Q)^{c}}\left(T * k^{j}\right)(z)\left(\Delta^{k} \partial_{j} g * \frac{1}{|y|^{n-\alpha}}\right)(z) d z \\
& +\sum_{j=1}^{n} c_{n, \alpha} \int_{3 Q}\left(T * k^{j}\right)(z)\left(\Delta^{k} \partial_{j} g * \frac{1}{|y|^{n-\alpha}}\right)(z) d z \equiv A+B .
\end{aligned}
$$

To deal with $A$ we use that $T * k^{j}$ is a bounded function. Notice that for $x \in(3 Q)^{c}$ and $y \in 2 Q$ we have

$$
|g(y)| \leq \frac{C\left\|\varphi_{Q}\right\|_{\infty}}{\ell(Q)^{\alpha}}
$$

Let $Q_{0}$ stand for the unit cube centered at 0 . Moving $\Delta^{k} \partial_{j}$ from $g$ to $\frac{1}{|y|^{n-\alpha}}$ and making the obvious change of variables one gets

$$
\begin{aligned}
& |A| \leq C \sup _{1 \leq i \leq n}\left\|T * k^{i}\right\|_{\infty} \frac{\left\|\varphi_{Q}\right\|_{\infty}}{\ell(Q)^{\alpha}} \int_{(3 Q)^{c}} \int_{2 Q} \frac{d y d z}{|z-y|^{2 n-\alpha}} \\
& \leq C \sup _{1 \leq i \leq n}\left\|T * k^{i}\right\|_{\infty} \int_{\left(3 Q_{0}\right)^{c}} \int_{2 Q_{0}} \frac{d y d z}{|z-y|^{2 n-\alpha}} \leq C \sup _{1 \leq i \leq n}\left\|T * k^{i}\right\|_{\infty} .
\end{aligned}
$$

Let's now turn our attention to $B$. Recall that we have

$$
\Delta^{k}(h g)=\sum_{i_{1}, \ldots, i_{k}=1}^{n} \sum_{l_{1}, \ldots, l_{k}=0}^{2}\left(\begin{array}{l}
2 \\
l_{1}
\end{array}\right) \ldots\left(\begin{array}{l}
2 \\
l_{k}
\end{array}\right) \partial_{i_{1} \ldots i_{k}}^{l_{1} \ldots l_{k}} h \partial_{i_{1} \ldots i_{k}}^{2-l_{1} \ldots 2-l_{k}} g
$$

where $\partial_{i_{1} \ldots i_{k}}^{l_{1} \ldots l_{k}}=\left(\partial_{i_{1}}\right)^{l_{1}} \ldots\left(\partial_{i_{k}}\right)^{l_{k}}$.

Since

$$
\Delta^{k}\left(\partial_{j} g\right)=\Delta^{k}\left(k_{x}^{i} \partial_{j} \varphi_{Q}\right)+\Delta^{k}\left(\varphi_{Q} \partial_{j} k_{x}^{i}\right)
$$


we have

$$
\begin{aligned}
& B \leq C \sup _{1 \leq i \leq n}\left\|T * k^{i}\right\|_{\infty} \int_{3 Q}\left|\left(\Delta^{k}\left(k_{x}^{i} \partial_{j} \varphi_{Q}\right) * \frac{1}{|y|^{n-\alpha}}\right)(z)\right| d z \\
& +C \sup _{1 \leq i \leq n}\left\|T * k^{i}\right\|_{\infty} \int_{3 Q}\left|\left(\Delta^{k}\left(\varphi_{Q} \partial_{j} k_{x}^{i}\right) * \frac{1}{|y|^{n-\alpha}}\right)(z)\right| d z \\
& \equiv C \sup _{1 \leq i \leq n}\left\|T * \frac{x_{i}}{|x|^{1+\alpha}}\right\|_{\infty}\left(B_{1}+B_{2}\right) .
\end{aligned}
$$

Using (8), support $\varphi_{Q} \subset 2 Q,\left\|\partial^{s} \varphi_{Q}\right\|_{\infty} \leq C_{s} \ell(Q)^{-|s|},|s| \geq 0, x \notin 3 Q$ and changing variables, we get

$$
\begin{aligned}
& B_{1} \leq \sum_{i_{1}, \ldots, i_{k}=1}^{n} \sum_{l_{1}, \ldots, l_{k}=0}^{2} \frac{C}{\ell(Q)^{l_{1}+\ldots+l_{k}+1}} \int_{3 Q} \int_{2 Q} \frac{d z d y}{|z-y|^{n-\alpha}|x-y|^{\alpha+2-l_{1}+\ldots+2-l_{k}}} \\
& \leq \frac{C}{\ell(Q)^{n+\alpha}} \int_{3 Q} \int_{2 Q} \frac{d z d y}{|z-y|^{n-\alpha}}=\frac{C \ell(Q)^{2 n}}{\ell(Q)^{n+\alpha+n-\alpha}} \int_{3 Q_{0}} \int_{2 Q_{0}} \frac{d z d y}{|z-y|^{n-\alpha}} \\
& \leq C .
\end{aligned}
$$

Arguing similarly we obtain $B_{2} \leq C$ and therefore we conclude that

$$
A+B \leq C \sup _{1 \leq i \leq n}\left\|T * k^{i}\right\|_{\infty}
$$

Case 2: $x \in 3 Q$. Without loss of generality assume $x=0$. Now the function $g(y)=$ $-\varphi_{Q}(y) k^{i}(y)$ may not be smooth, but (6) still holds in the distributions sense. In fact, a different version of (6) will be used for this case. Since $\alpha$ is noninteger and $[\alpha]=2 d$ we readily get

$$
f=C \sum_{j=1}^{n} \Delta^{k-d} \partial_{j} f * \frac{1}{|x|^{n-\alpha+2 d}} * \frac{x_{j}}{|x|^{1+\alpha}}
$$

where $C=C(n, \alpha)$ and the above identity holds in the distributions sense.

Define $f=T * \frac{1}{|x|^{\alpha-1}}$. Since $\partial_{j} f=C\left(T * k^{j}\right)$ and the $T * k^{j}$ are bounded, the function $f$ satisfies a Lipschitz condition of order 1 . We get 


$$
\begin{aligned}
& \left(\varphi_{Q} T * k^{i}\right)(0)=<T, g>=c_{n, \alpha} \sum_{j=1}^{n}<T * k^{j}, \Delta^{k-d} \partial_{j} g * \frac{1}{|y|^{n-\alpha+2 d}}> \\
& =C \sum_{j=1}^{n}<\partial_{j}(f-f(0)), \Delta^{k-d} \partial_{j} g * \frac{1}{|y|^{n-\alpha+2 d}}>.
\end{aligned}
$$

We claim now that integrating by parts gives

$$
\begin{aligned}
& \sum_{j=1}^{n}<\partial_{j}(f-f(0)), \Delta^{k-d} \partial_{j} g * \frac{1}{|y|^{n-\alpha+2 d}}> \\
& =<f-f(0), \Delta^{k-d+1} g * \frac{1}{|y|^{n-\alpha+2 d}}>+O\left(\sup _{1 \leq i \leq n}\left\|T * k^{i}\right\|_{\infty}\right) .
\end{aligned}
$$

We postpone the proof of (11) and we continue with the argument. If (11) holds, then we can write

$$
\begin{aligned}
& \left|\left(\varphi_{Q} T * k^{i}\right)(0)\right| \leq C\left|\int_{(3 Q)^{c}}(f(z)-f(0))\left(\Delta^{k+1-d} g * \frac{1}{|y|^{n-\alpha+2 d}}\right)(z) d z\right| \\
& +C\left|\int_{3 Q}(f(z)-f(0))\left(\Delta^{k+1-d} g * \frac{1}{|y|^{n-\alpha+2 d}}\right)(z) d z\right|+C \sup _{1 \leq i \leq n}\left\|T * k^{i}\right\|_{\infty} .
\end{aligned}
$$

Set

$$
A=\int_{(3 Q)^{c}}(f(z)-f(0))\left(\Delta^{k+1-d} g * \frac{1}{|y|^{n-\alpha+2 d}}\right)(z) d z
$$

and

$$
B=\int_{3 Q}(f(z)-f(0))\left(\Delta^{k+1-d} g * \frac{1}{|y|^{n-\alpha+2 d}}\right)(z) d z .
$$

Using the boundedness of the function $T * k^{j}=\partial_{j} f$, Fubini and changing variables we obtain 


$$
\begin{aligned}
& |A| \leq C \sup _{1 \leq i \leq n}\left\|T * k^{i}\right\|_{\infty} \sum_{j=1}^{n} \int_{(3 Q)^{c}}|z| \int_{2 Q} \frac{|g(y)|}{|z-y|^{2 n+1-\alpha}} d y d z \\
& \leq C \sup _{1 \leq i \leq n}\left\|T * k^{i}\right\|_{\infty}\left\|\varphi_{Q}\right\|_{\infty} \sum_{j=1}^{n} \int_{(3 Q)^{c}} \int_{2 Q} \frac{|z-y|+|y|}{|y|^{\alpha}|z-y|^{2 n+1-\alpha}} d y d z \\
& \leq C \sup _{1 \leq i \leq n}\left\|T * k^{i}\right\|_{\infty} \sum_{j=1}^{n} \int_{2 Q} \frac{1}{|y|^{\alpha}} \int_{(3 Q)^{c}} \frac{d z}{|z-y|^{2 n-\alpha}} d y \\
& +C \sup _{1 \leq i \leq n}\left\|T * k^{i}\right\|_{\infty} \sum_{j=1}^{n} \ell(Q) \int_{2 Q} \frac{1}{|y|^{\alpha}} \int_{(3 Q)^{c}} \frac{d z}{|z-y|^{2 n+1-\alpha}} d y \\
& \leq C \sup _{1 \leq i \leq n}\left\|T * k^{i}\right\|_{\infty} .
\end{aligned}
$$

For the term $B$, write

$$
\begin{aligned}
& |B|=\left|\int_{3 Q}(f(z)-f(0))\left(\Delta^{k+1-d} g * \frac{1}{|y|^{n-\alpha+2 d}}\right)(z) d z\right| \\
& \leq C\left|\int_{3 Q} \sum_{|r|+|s|=n+1-2 d}(f(z)-f(0))\left(\left(\partial^{r} \varphi_{Q} \partial^{s} k^{i}\right) * \frac{1}{|y|^{n-\alpha+2 d}}\right)(z) d z\right|,
\end{aligned}
$$

where the last sum is over those multi-indexes $r$ and $s$ that appear in distributing between $\varphi_{Q}$ and $k^{i}$ the $n+1-2 d$ derivatives coming from $\Delta^{k+1-d}$. We will now divide the above sum in two parts, the first one containing the indexes $|r| \geq 2$ and the second one the remaining indexes. In order to be able to estimate the integral of this second part, which is the worse, we will have to subtract a Taylor polynomial of $\varphi_{Q}$ of order one. Let

$$
R(y)=\varphi_{Q}(y)-\sum_{|m|=0}^{1} \partial^{m} \varphi_{Q}(0) y^{m}
$$


Then

$$
\begin{aligned}
& |B| \leq C \sum_{|r| \geq 2} \int_{3 Q}|f(z)-f(0)| \int_{2 Q} \frac{d y d z}{\ell(Q)^{|r|}|y|^{\alpha+n+1-2 d-|r|}|z-y|^{n-\alpha+2 d}} \\
& +C \int_{3 Q}|f(z)-f(0)|\left|\sum_{\substack{|r|+|s|=n+1-2 d \\
|r| \leq 1}} \int \frac{\partial^{r} R(y) \partial^{s} k^{i}(y)}{|z-y|^{n-\alpha+2 d}} d y\right| d z \\
& +C\left|\int_{3 Q}(f(z)-f(0)) \sum_{\substack{|m|=0 \\
1}} \partial^{m} \varphi(0)\left(y^{m} \Delta^{k+1-d} k^{i} * \frac{1}{|y|^{n-\alpha+2 d}}\right)(z) d z\right| \\
& +C \sup _{|m|=1}\left|\partial^{m} \varphi_{Q}(0)\right|\left|\int_{3 Q}(f(z)-f(0)) \sum_{|s|=n-2 d}\left(\partial^{s} k^{i} * \frac{1}{|y|^{n-\alpha+2 d}}\right)(z) d z\right| \\
& \equiv B_{1}+B_{2}+B_{3}+B_{4} .
\end{aligned}
$$

Notice that if $|r| \geq 2$, then we have $\alpha+n+1-2 d-|r| \leq \alpha+n-1-2 d<n$. Hence using the boundedness of the functions $T * k^{i}, 1 \leq i \leq n$, we conclude that $B_{1}$ is finite and, by homogeneity, independent of $\ell(Q)$. Thus,

$$
B_{1} \leq C \sup _{1 \leq i \leq n}\left\|T * k^{i}\right\|_{\infty}
$$

We deal now with $B_{2}$. Write

$$
\begin{aligned}
& B_{2}=C \int_{3 Q}|f(z)-f(0)|\left|\sum_{\substack{|r|+|s|=n+1-2 d \\
|r| \leq 1}} \int_{4 Q} \frac{\partial^{r} R(y) \partial^{s} k^{i}(y)}{|z-y|^{n-\alpha+2 d}} d y\right| d z \\
& +C \int_{3 Q}|f(z)-f(0)|\left|\sum_{\substack{|r|+|s|=n+1-2 d \\
|r| \leq 1}} \int_{(4 Q)^{c}} \frac{\partial^{r} R(y) \partial^{s} k^{i}(y)}{|z-y|^{n-\alpha+2 d}} d y\right| d z=B_{21}+B_{22} .
\end{aligned}
$$

For the integral over $4 Q$, we have to use the Taylor expansion to get integrability. For the terms with $|r|=1$ we use that

$$
\left|\partial^{r} R(y)\right|=\left|\partial^{r} \varphi_{Q}(y)-\partial^{r} \varphi_{Q}(0)\right| \leq C \frac{|y|}{\ell(Q) 2}
$$


and for the term with $|r|=0$

$$
|R(y)| \leq C \frac{|y|^{2}}{\ell(Q) 2}
$$

Therefore

$$
\begin{aligned}
& B_{21} \leq C \sup _{1 \leq i \leq n}\left\|T * k^{i}\right\|_{\infty} \ell(Q) \int_{3 Q} \int_{4 Q} \frac{|y|}{\ell(Q) 2|y|^{\alpha+n-2 d}|z-y|^{n-\alpha+2 d}} d y d z \\
& +C \sup _{1 \leq i \leq n}\left\|T * k^{i}\right\|_{\infty} \ell(Q) \int_{3 Q} \int_{4 Q} \frac{|y|^{2}}{\ell(Q) 2|y|^{\alpha+n-2 d-1}|z-y|^{n-\alpha+2 d}} d y d z . \\
& \leq C \sup _{1 \leq i \leq n}\left\|T * k^{i}\right\|_{\infty} \ell(Q)^{-1} \int_{3 Q} \int_{4 Q} \frac{d y d z}{|y|^{\alpha+n-2 d-1|z-y|^{n-\alpha+2 d}} .}
\end{aligned}
$$

Then by homogeneity and local integrability,

$$
B_{21} \leq C \sup _{1 \leq i \leq n}\left\|T * k^{i}\right\|_{\infty} .
$$

For the integral over $(4 Q)^{c}$, we do not apply Taylor's formula; we just estimate term by term. For $|r|=0$ (and then $|s|=n+1-2 d$ ) we have that

$$
\left|R(y) \partial^{s} k^{i}(y)\right| \leq \frac{C|y|}{\ell(Q)|y|^{\alpha+n+1-2 d}}=\frac{C}{\ell(Q)|y|^{n+\alpha-2 d}} .
$$

For $|r|=1$ the term $\left|\partial^{r} R(y) \partial^{s} k^{i}(y)\right|$ can be estimated by $C \ell(Q)^{-1}|y|^{-\alpha-n+2 d}$, because now $|s|=n-2 d$. Therefore

$$
\begin{aligned}
& B_{22} \leq C \sup _{1 \leq i \leq n}\left\|T * k^{i}\right\|_{\infty} \ell(Q) \int_{3 Q} \int_{(4 Q)^{c}} \frac{d y}{\ell(Q)|y|^{\alpha+n-2 d}|z-y|^{n-\alpha+2 d}} d z \\
& \leq C \sup _{1 \leq i \leq n}\left\|T * k^{i}\right\|_{\infty} .
\end{aligned}
$$

For $B_{3}$, separate the terms according to whether $|m|=0$ or $|m|=1$ as follows:

$$
\begin{aligned}
& B_{3}=\left|\int_{3 Q}(f(z)-f(0)) \varphi_{Q}(0)\left(\Delta^{k+1-d} k^{i} * \frac{1}{|y|^{n-\alpha+2 d}}\right)(z) d z\right| \\
& +\left|\int_{3 Q}(f(z)-f(0)) \sum_{|m|=1} \partial^{m} \varphi_{Q}(0)\left(y^{m} \Delta^{k+1-d} k^{i} * \frac{1}{|y|^{n-\alpha+2 d}}\right)(z) d z\right| \\
& \equiv B_{31}+B_{32} .
\end{aligned}
$$


Now we treat the term $B_{31}$. Taking Fourier transforms on the convolution $\Delta^{k+1-d} k^{i} * \frac{1}{|y|^{n-\alpha+2 d}}$ we obtain for an appropriate constant $C$,

$$
\left(\Delta^{k+1-d} k^{i} * \frac{1}{|y|^{n-\alpha+2 d}}\right)(\xi)=C \xi_{i}
$$

Thus

$$
\Delta^{k+1-d} k^{i} * \frac{1}{|y|^{n-\alpha+2 d}}=C \partial_{i} \delta
$$

Hence, by a standard regularization process that we omit,

$$
\begin{aligned}
B_{31} & =C\left|\varphi_{Q}(0)<\partial_{i} \delta, f(z)-f(0)>\right|=C\left|\varphi_{Q}(0)<\delta, \partial_{i} f>\right|=C\left|\varphi_{Q}(0) \partial_{i} f(0)\right| \\
& \leq C\left\|\varphi_{Q}\right\|_{\infty} \sup _{1 \leq i \leq n}\left\|T * k^{i}\right\|_{\infty} .
\end{aligned}
$$

To estimate $B_{32}$, we take the Fourier transform of $y^{m} \Delta^{k+1-d} k^{i} * \frac{1}{|y|^{n-\alpha+2 d}},|m|=$ 1. We get

$$
\left(y^{m} \Delta^{k+1-d} k^{i} * \frac{1}{|y|^{n-\alpha+2 d}}\right)(\xi)=C \partial^{m}\left(\frac{|\xi|^{2 k+2-2 d} \xi_{i}}{|\xi|^{1+n-\alpha}}\right) \frac{1}{|\xi|^{\alpha-2 d}}=C \delta_{m, m_{i}}+C \frac{\xi^{m} \xi_{i}}{|\xi|^{2}}
$$

where $m_{i}$ is the multi-index with all entries equal to 0 except the $i$-th entry which is $1 ; \delta_{m, m_{i}}$ equals one when $m=m_{i}$ and zero otherwise. Hence

$$
y^{m} \Delta^{k+1-d} k^{i} * \frac{1}{|y|^{n-\alpha+2 d}}=C \delta_{m, m_{i}} \delta+C \text { P.V. } \frac{z^{m} z_{i}}{|z|^{n+2}},
$$

where P.V. stands for principal value. Since $|m|=1$,

$$
\begin{aligned}
B_{32} & =\left|\sum_{|m|=1} \partial^{m} \varphi_{Q}(0) \int_{3 Q}(f(z)-f(0)) \frac{z^{m} z_{i}}{|z|^{n+2}}\right| \leq \frac{C}{\ell(Q)} \sup _{1 \leq i \leq n}\left\|T * k^{i}\right\|_{\infty} \int_{3 Q} \frac{d z}{|z|^{n-1}} \\
& \leq C \sup _{1 \leq i \leq n}\left\|T * k^{i}\right\|_{\infty} .
\end{aligned}
$$

Now we are left with term $B_{4}$. Taking Fourier transforms on the convolution $\partial^{s} k^{i} * \frac{1}{|y|^{n-\alpha+2 d}}$, we obtain 


$$
\left(\partial^{s} k^{i} * \frac{1}{|y|^{n-\alpha+2 d}}\right)(\xi)=C \xi^{s} \frac{\xi_{i}}{|\xi|^{1+n-\alpha}} \frac{1}{|\xi|^{\alpha-2 d}}=C \frac{\xi^{s} \xi_{i}}{|\xi|^{n+1-2 d}} .
$$

Hence, since $|s|=n-2 d$,

$$
\partial^{s} k^{i} * \frac{1}{|y|^{n-\alpha}}=C P . V \cdot \frac{z^{s} z_{i}}{|z|^{2|s|+1+2 d}} .
$$

Arguing as before

$$
B_{4} \leq C \sup _{1 \leq i \leq n}\left\|T * k^{i}\right\|_{\infty} \frac{1}{\ell(Q)} \int_{3 Q} \frac{d z}{|z|^{n-1}} \leq C \sup _{1 \leq i \leq n}\left\|T * k^{i}\right\|_{\infty} .
$$

We still have to show claim (111). Let $1 \leq j \leq n$ and set

$$
\omega_{j}=(-1)^{j-1} d y_{1} \wedge \cdots \wedge \widehat{d y}_{j} \wedge \cdots \wedge d y_{n} .
$$

Then, the Green-Stokes Theorem gives

$$
\begin{aligned}
& \sum_{j=1}^{n}<\partial_{j}(f-f(0)), \Delta^{k-d} \partial_{j} g * \frac{1}{|y|^{n-\alpha+2 d}}> \\
& =-<f-f(0), \Delta^{k-d+1} g * \frac{1}{|y|^{n-\alpha+2 d}}> \\
& +\sum_{j=1}^{n} \lim _{\varepsilon \rightarrow 0} \int_{|y|=\varepsilon^{-1}}(f(y)-f(0))\left(g * \Delta^{k-d} \partial_{j} \frac{1}{|y|^{n-\alpha+2 d}}\right)(y) \omega_{j} \\
& -\sum_{j=1}^{n} \lim _{\varepsilon \rightarrow 0} \int_{|y|=\varepsilon}(f(y)-f(0))\left(\Delta^{k-d} \partial_{j} g * \frac{1}{|y|^{n-\alpha+2 d}}\right)(y) \omega_{j} .
\end{aligned}
$$

The integral over the sphere of radius $\epsilon^{-1}$ can be easily estimated by a constant times $\varepsilon^{n-\alpha}$. Thus we are only left with the integral over the sphere of radius $\epsilon$. For $1 \leq j \leq n$ and for a suitable constant $C$ we can write (recall that for some constant $C$ depending on $n$ and $\left.\alpha, \frac{C}{|y|^{n-\alpha+2 d}}=\Delta\left(\frac{1}{|y|^{n-\alpha+2 d-2}}\right)\right)$

$$
\begin{aligned}
& \int_{|y|=\varepsilon}(f(y)-f(0))\left(\Delta^{k-d} \partial_{j} g * \frac{1}{|y|^{n-\alpha+2 d}}\right)(y) \omega_{j} \\
& =C \sum_{l=0}^{n} \int_{|y|=\varepsilon}(f(y)-f(0))\left(\Delta^{k-d} \partial_{j} \partial_{l} g * \frac{y_{l}}{|y|^{n-\alpha+2 d}}\right)(y) \omega_{j} .
\end{aligned}
$$


Notice that when looking at the above integral, the worst case arises when all the derivatives $\Delta^{k-d} \partial_{j} \partial_{l}$ of the product $g=-\varphi_{Q} k^{i}$ are taken on the factor $k^{i}$. We will only be concerned with this case. For the other cases argue like in (12). Recall that $R(y)=\varphi_{Q}(y)-\sum_{|m|=0}^{1} \partial^{m} \varphi_{Q}(0) y^{m}$. To get integrability we use the Taylor expansion of $\varphi_{Q}$ up to order 1 . Then for $1 \leq j \leq n$ we have

$$
\begin{aligned}
& \int_{|y|=\varepsilon}(f(y)-f(0))\left(\sum_{l} \varphi_{Q} \Delta^{k-d} \partial_{j} \partial_{l} k^{i} * \frac{y_{l}}{|y|^{n-\alpha+2 d}}\right)(y) \omega_{j} \\
& =\int_{|y|=\varepsilon}(f(y)-f(0))\left(\sum_{l} R \Delta^{k-d} \partial_{j} \partial_{l} k^{i} * \frac{y_{l}}{|y|^{n-\alpha+2 d}}\right)(y) \omega_{j} \\
& +\varphi_{Q}(0) \int_{|y|=\varepsilon}(f(y)-f(0))\left(\sum_{l} \Delta^{k-d} \partial_{j} \partial_{l} k^{i} * \frac{y_{l}}{|y|^{n-\alpha+2 d}}\right)(y) \omega_{j} \\
& +\sum_{|m|=1} \partial^{m} \varphi_{Q}(0) \int_{|y|=\varepsilon}(f(y)-f(0)) \sum_{l}\left(y^{m} \Delta^{k-d} \partial_{j} \partial_{l} k^{i} * \frac{y_{l}}{|y|^{n-\alpha+2 d}}\right)(y) \omega_{j} \\
& =A_{1}+A_{2}+A_{3} .
\end{aligned}
$$

We will now show that $A_{1}$ and $A_{3}$ converge to zero when $\varepsilon \rightarrow 0$ and that $A_{2}$ is bounded above by $C \sup _{1 \leq i \leq n}\left\|T * k^{i}\right\|_{\infty}$.

For $A_{1}$ we break the convolution integral into two terms corresponding to $3 Q$ and $(3 Q)^{c}$ :

$$
\begin{aligned}
& A_{1}=\int_{|y|=\varepsilon}(f(y)-f(0)) \int_{3 Q} R(z) \sum_{l} \Delta^{k-d} \partial_{j} \partial_{l} k^{i}(z) \frac{y_{l}-z_{l}}{|y-z|^{n-\alpha+2 d}} d z \omega_{j} \\
& +\int_{|y|=\varepsilon}(f(y)-f(0)) \int_{(3 Q)^{c}} R(z) \sum_{l} \Delta^{k-d} \partial_{j} \partial_{l} k^{i}(z) \frac{y_{l}-z_{l}}{|y-z|^{n-\alpha+2 d}} d z \omega_{j} \\
& =A_{11}+A_{12} .
\end{aligned}
$$

We deal first with $A_{11}$. Since $|R(z)| \leq C|z|^{2} \ell(Q)^{-2}$, the product $R \Delta^{k-d} \partial_{j} \partial_{l} k^{i}$ is a locally integrable function. Thus, using the boundedness of $T * k^{j}$, we get

$$
\left|A_{11}\right| \leq C \varepsilon \int_{|y|=\varepsilon} \int_{3 Q} \frac{d z}{|z|^{n-1+\alpha-2 d}|z-y|^{n-\alpha+2 d-1}}\left|\omega_{j}\right| \leq C \varepsilon^{1+\alpha-2 d} .
$$

Since we also have $|R(z)| \leq c|z| \ell(Q)^{-1}$, we obtain 


$$
\left|A_{12}\right| \leq C \varepsilon \int_{|y|=\varepsilon} \int_{(3 Q)^{c}} \frac{d z}{|z|^{n+\alpha-2 d}|z-y|^{n-\alpha+2 d-1}}\left|\omega_{j}\right| \leq C \varepsilon^{n} .
$$

Thus $A_{1}$ tends to zero with $\varepsilon$.

To estimate $A_{2}$, take the Fourier transforms of $\sum_{l} \Delta^{k-d} \partial_{j} \partial_{l} k^{i} * \frac{y_{l}}{|y|^{n-\alpha+2 d}}$. Then for an appropriate constant $C$ one has

$$
\left(\sum_{l} \Delta^{k-d} \partial_{j} \partial_{l} k^{i} * \frac{y_{l}}{|y|^{n-\alpha+2 d}}\right)(\xi)=C \sum_{l}|\xi|^{2 k-2 d} \xi_{j} \xi_{l} \frac{\xi_{i}}{|\xi|^{1+n-\alpha}} \frac{\xi_{l}}{|\xi|^{2+\alpha-2 d}}=C \frac{\xi_{i} \xi_{j}}{|\xi|^{2}}
$$

Thus

$$
\sum_{l} \Delta^{k-d} \partial_{j} \partial_{l} k^{i} * \frac{y_{l}}{|y|^{n-\alpha+2 d}}=C \text { P.V. } \frac{y_{i} y_{j}}{|y|^{n+2}}
$$

Hence

$$
\begin{aligned}
& \left|A_{2}\right|=\left|C \varphi_{Q}(0) \int_{|y|=\varepsilon}(f(y)-f(0)) \frac{y_{i} y_{j}}{|y|^{n+2}} \omega_{j}\right| \\
& \leq C \sup _{1 \leq i \leq n}\left\|T * k^{i}\right\|_{\infty} \varepsilon^{1-n} \int_{|y|=\varepsilon}\left|\omega_{j}\right|=C \sup _{1 \leq i \leq n}\left\|T * k^{i}\right\|_{\infty} .
\end{aligned}
$$

For the last term $A_{3}$, taking the Fourier transform of $\sum_{l} y^{m} \Delta^{k-d} \partial_{j} \partial_{l} k^{i} *$ $\frac{y_{l}}{|y|^{\mid-\alpha+2 d}}$, we get for a suitable constants $C_{1}$ and $C_{2}$

$$
\begin{aligned}
& \left(\sum_{l} y^{m} \Delta^{k-d} \partial_{j} \partial_{l} k^{i} * \frac{y_{l}}{|y|^{n-\alpha+2 d}}\right)(\xi) \\
& =C_{1} \sum_{l} \partial^{m}\left(\frac{|\xi|^{2 k-2 d} \xi_{j} \xi_{l} \xi_{i}}{|\xi|^{1+n-\alpha}}\right) \frac{\xi_{l}}{|\xi|^{2+\alpha-2 d}}=C_{1} \sum_{l} \partial^{m}\left(\frac{\xi_{j} \xi_{l} \xi_{i}}{|\xi|^{2+2 d-\alpha}}\right) \frac{\xi_{l}}{|\xi|^{2+\alpha-2 d}} \\
& =C_{1} \sum_{l}\left(\delta_{m, m_{j}} \frac{\xi_{i} \xi_{l}}{|\xi|^{2+2 d-\alpha}}+\delta_{m, m_{i}} \frac{\xi_{j} \xi_{l}}{|\xi|^{2+2 d-\alpha}}+\delta_{m, m_{l}} \frac{\xi_{i} \xi_{j}}{|\xi|^{2+2 d-\alpha}}+C_{2} \frac{\xi_{j} \xi_{i} \xi_{l} \xi^{m}}{|\xi|^{4+2 d-\alpha}}\right) \frac{\xi_{l}}{|\xi|^{\alpha+2-2 d}} \\
& =C_{1}\left(\delta_{m, m_{j}} \frac{\xi_{i}}{|\xi|^{2}}+\delta_{m, m_{i}} \frac{\xi_{j}}{|\xi|^{2}}+C_{2} \frac{\xi^{m} \xi_{j} \xi_{i}}{|\xi|^{4}}+\sum_{l} \delta_{m, m_{l}} \frac{\xi_{i} \xi_{j} \xi_{l}}{|\xi|^{4}}\right) .
\end{aligned}
$$


Hence

$$
\begin{aligned}
& \left(\sum_{l} y^{m} \Delta^{k-d} \partial_{j} \partial_{l} k^{i} * \frac{y_{l}}{|y|^{n-\alpha+2 d}}\right)(y) \\
& =C_{3}\left(\delta_{m, m_{j}} \frac{y_{i}}{|y|^{n}}+\delta_{m, m_{i}} \frac{y_{j}}{|y|^{n}}+C_{2} \frac{y^{m} y_{j} y_{i}}{|y|^{n+2}}+\sum_{l} \delta_{m, m_{l}} \frac{y_{i} y_{j} y_{l}}{|y|^{n+2}}\right)
\end{aligned}
$$

and since $|m|=1$,

$$
\left|A_{3}\right| \leq C \int_{|y|=\varepsilon} \frac{|f(y)-f(0)|}{|y|^{n-1}}\left|\omega_{j}\right| \leq C \varepsilon^{2-n} \int_{|y|=\varepsilon}\left|\omega_{j}\right|=C \varepsilon,
$$

which completes the proof of claim (11).

If $n$ is odd, $\alpha$ non-integer, but $[\alpha]=2 d+1$ is also odd, then we replace the reproducing formula (10) by

$$
f=C \sum_{j=1}^{n} \Delta^{k-d} f * \frac{x_{j}}{|x|^{n-\alpha+2 d+2}} * \frac{x_{j}}{|x|^{1+\alpha}} .
$$

If $n$ is odd and $\alpha$ is an odd integer of the form $\alpha=2 d+1$, then we use the reproducing formula (10). Instead of applying Taylor's expansion up to order 1, we need in this case to apply Taylor's expansion up to order 2.

If $n$ is odd and $\alpha$ is an even integer of the form $\alpha=2 d+2$ we use again formula (13) and Taylor's expansion up to order 2.

If $n$ is even we use suitable reproducing formulas (see Lemma 11 in $[\mathrm{P}]$ ) and Taylor's expansions up to order 1 if $\alpha$ is non-integer and up to order 2 if $\alpha$ is integer.

\section{Proof of the Theorem.}

Let $\mu$ be a positive Radon measure and $0<\alpha<1$. For $x \in \mathbb{R}^{n}$, set

$$
\begin{aligned}
& p_{\alpha} 2(\mu)(x)=\int_{\mathbb{R}^{n}} \int_{\mathbb{R}^{n}} p_{\alpha}(x, y, z) d \mu(y) d \mu(z), \\
& M_{\alpha} \mu(x)=\sup _{r>0} \frac{\mu(B(x, r))}{r^{\alpha}}
\end{aligned}
$$

and 


$$
U_{\alpha}^{\mu}(x)=M_{\alpha} \mu(x)+p_{\alpha}(\mu)(x) .
$$

Recall that in section 2 we defined $p_{\alpha}(\mu)=\int_{\mathbb{R}^{n}} \int_{\mathbb{R}^{n}} \int_{\mathbb{R}^{n}} p_{\alpha}(x, y, z) d \mu(x) d \mu(y) d \mu(z)$. Observe that $p_{\alpha}(\mu)=\int_{\mathbb{R}^{n}} p_{\alpha} 2(\mu)(x) d \mu(x)$. $U_{\alpha}^{\mu}$ is the analogue of the potential introduced in $\mathrm{V} 2$. The energy associated to this potential is

$$
E_{\alpha}(\mu)=\int_{\mathbb{R}^{n}} U_{\alpha}^{\mu}(x) d \mu(x) .
$$

Lemma 4.1. For each compact set $K \subset \mathbb{R}^{n}$ and $0<\alpha<1$ we have

$$
\gamma_{\alpha,+}(K) \approx \sup _{\nu} \frac{1}{E_{\alpha}(\nu)}
$$

where the supremum is taken over the probability measures $\nu$ supported on $K$.

Proof. Take a positive Radon measure $\mu$ supported on $K$ such that $\left|\left(\frac{x_{i}}{|x|^{1+\alpha}} * \mu\right)(x)\right| \leq$ 1 for almost all $x \in \mathbb{R}^{n}, 1 \leq i \leq n$. We claim that

$$
\mu(B(x, r)) \leq C r^{\alpha}, \quad x \in \mathbb{R}^{n}, \quad r>0 .
$$

To prove the claim take an infinitely differentiable function $\varphi$, supported on $B(x, 2 r)$ such that $\varphi=1$ on $B(x, r)$, and $\left\|\partial^{s} \varphi\right\|_{\infty} \leq C_{s} r^{-|s|},|s| \geq 0$. Assume first that $n$ is odd and of the form $n=2 k+1$. Then, by Lemma 11 in $[\mathrm{P}]$,

$$
\begin{aligned}
& \mu(B(x, r)) \leq \int \varphi d \mu=c_{n, \alpha} \int\left(\sum_{i=1}^{n} \Delta^{k} \partial_{i} \varphi * \frac{1}{|x|^{n-\alpha}} * \frac{x_{i}}{|x|^{1+\alpha}}\right)(y) d \mu(y) \\
& =-c_{n, \alpha} \sum_{i=1}^{n} \int\left(\mu * \frac{x_{i}}{|x|^{1+\alpha}}\right)(y)\left(\Delta^{k} \partial_{i} \varphi * \frac{1}{|x|^{n-\alpha}}\right)(y) d y \\
& \leq C \sum_{i=1}^{n}\left(\int_{B(x, 3 r)}\left|\left(\Delta^{k} \partial_{i} \varphi * \frac{1}{|x|^{n-\alpha}}\right)(y)\right| d y+\int_{\mathbb{R}^{n} \backslash B(x, 3 r)}\left|\left(\Delta^{k} \partial_{i} \varphi * \frac{1}{|x|^{n-\alpha}}\right)(y)\right| d y\right) .
\end{aligned}
$$

Arguing as in Lemma 3.1 we get that the last two integrals can be estimated by $\mathrm{Cr}^{\alpha}$

If $n$ is even we use the corresponding representation formula in Lemma 11 of $[\mathrm{P}$.

On the other hand, it can be easily shown that

$$
\left|R_{\alpha, \varepsilon}(\mu)(x)\right| \leq C, \quad x \in \mathbb{R}^{n}, \varepsilon>0,
$$

and so, by (41), we obtain 


$$
p_{\alpha}(\mu) \leq C\|\mu\|
$$

By Schwarz inequality

$$
E_{\alpha}(\mu) \leq C\|\mu\|+\|\mu\|^{1 / 2} p_{\alpha}(\mu)^{1 / 2} \leq C\|\mu\|
$$

Set $\nu=\mu /\|\mu\|$, so that

$$
E_{\alpha}(\nu)=\frac{E_{\alpha}(\mu)}{\|\mu\|^{2}} \leq \frac{C}{\|\mu\|}
$$

and consequently

$$
\gamma_{\alpha,+}(K) \leq C \sup _{\nu} \frac{1}{E_{\alpha}(\nu)}
$$

The reverse inequality is proved as in $\mathrm{V2}$ and involves the $T(1)$-Theorem for non-doubling measures.

It is a crucial fact that the capacity $C_{s, p}$ can be described by means of Wolff potentials. The Wolff potential of a positive Radon measure $\mu$ is defined by

$$
W^{\mu}(x)=W_{s, p}^{\mu}(x)=\int_{0}^{\infty}\left(\frac{\mu(B(x, r))}{r^{n-s p}}\right)^{p^{\prime}-1} \frac{d r}{r}, x \in \mathbb{R}^{n},
$$

where $p^{\prime}=p /(p-1)$ is the exponent conjugate to $p$.

The Wolff energy of $\mu$ is

$$
E(\mu)=E_{s, p}(\mu)=\int_{\mathbb{R}^{n}} W^{\mu}(x) d \mu(x) .
$$

By Wolff's inequality ([AH], Theorem 4.5.4, p.110) one has

$$
C^{-1} \sup _{\mu} \frac{1}{E_{s, p}(\mu)^{p-1}} \leq C_{s, p}(K) \leq C \sup _{\mu} \frac{1}{E_{s, p}(\mu)^{p-1}},
$$

where $C$ is a positive constant depending only on $s, p$ and $n$, and the supremum is taken over the probability measures $\mu$ supported on $K$.

Lemma 4.2. For each positive finite Radon measure $\mu$ on $\mathbb{R}^{n}$ we have

$$
p_{\alpha}(\mu) \approx E_{\frac{2}{3}(n-\alpha), \frac{3}{2}}(\mu)=\int_{\mathbb{R}^{n}} \int_{0}^{\infty}\left(\frac{\mu(B(x, r))}{r^{\alpha}}\right) 2 \frac{d r}{r} d \mu(x) .
$$


Proof. Suppose that

$$
\int_{\mathbb{R}^{n}} \int_{0}^{\infty}\left(\frac{\mu(B(x, r))}{r^{\alpha}}\right) 2 \frac{d r}{r} d \mu(x)<\infty
$$

and set $G=\left\{\left(x_{1}, x_{2}, x_{3}\right):\left|x_{1}-x_{2}\right| \leq\left|x_{1}-x_{3}\right| \leq\left|x_{2}-x_{3}\right|\right\}$. Using Lemma 2.1 and Riemann-Stieltjes integration, we obtain

$$
\begin{aligned}
& p_{\alpha}(\mu)=3 \iiint_{G} p_{\alpha}\left(x_{1}, x_{2}, x_{3}\right) d \mu\left(x_{1}\right) d \mu\left(x_{2}\right) d \mu\left(x_{3}\right) \\
& \approx \iiint_{B\left(x_{3},\left|x_{2}-x_{3}\right|\right)}\left|x_{2}-x_{3}\right|^{-2 \alpha} d \mu\left(x_{1}\right) d \mu\left(x_{2}\right) d \mu\left(x_{3}\right) \\
& =\int_{\mathbb{R}^{n}} \int_{\mathbb{R}^{n}} \frac{\mu\left(B\left(x_{3},\left|x_{2}-x_{3}\right|\right)\right)}{\left|x_{2}-x_{3}\right|^{2 \alpha}} d \mu\left(x_{2}\right) d \mu\left(x_{3}\right) \\
& =\int_{\mathbb{R}^{n}} \int_{0}^{\infty} \frac{\mu\left(B\left(x_{3}, r\right)\right)}{r^{2 \alpha}} d \mu\left(B\left(x_{3}, r\right)\right) d \mu\left(x_{3}\right) .
\end{aligned}
$$

Notice that

$$
\lim _{r \rightarrow \infty}\left(\frac{\mu(B(x, r))}{r^{\alpha}}\right) 2 \leq \lim _{r \rightarrow \infty}\left(\frac{\mu\left(\mathbb{R}^{n}\right)}{r^{\alpha}}\right) 2=0 .
$$

Moreover,

$$
\int_{\rho}^{2 \rho}\left(\frac{\mu(B(x, r))}{r^{\alpha}}\right) 2 \frac{d r}{r} \geq \mu(B(x, \rho)) 2 \int_{\rho}^{2 \rho} \frac{d r}{r^{2 \alpha+1}}=C\left(\frac{\mu(B(x, \rho))}{\rho^{\alpha}}\right) 2 .
$$

Thus

$$
\lim _{r \rightarrow 0}\left(\frac{\mu(B(x, r))}{r^{\alpha}}\right) 2=0
$$

Integration by parts in the last integral of (14), together with (15) and (16), show that

$$
p_{\alpha}(\mu) \approx \int_{\mathbb{R}^{n}} \int_{0}^{\infty}\left(\frac{\mu(B(x, r))}{r^{\alpha}}\right) 2 \frac{d r}{r} d \mu(x) .
$$

Suppose now that $p_{\alpha}(\mu)<\infty$. We claim that we can assume that

$$
\liminf _{r \rightarrow 0} \frac{\mu(B(x, r))}{r^{\alpha}}=0, \text { for } \mu \text {-almost all } x \in \mathbb{R}^{n} .
$$


If (17) holds, then integrating by parts in the last integral of (14) one can deduce that

$$
p_{\alpha}(\mu) \approx \int_{\mathbb{R}^{n}} \int_{0}^{\infty}\left(\frac{\mu(B(x, r))}{r^{\alpha}}\right) 2 \frac{d r}{r} d \mu(x),
$$

and in this case we are done.

Otherwise there exists a $\mu$-measurable set $F$ such that $\mu(F)>0$ and

$$
\liminf _{r \rightarrow 0} \frac{\mu(B(x, r))}{r^{\alpha}}>0, \quad x \in F .
$$

Shrinking $F$ we can assume that

$$
\liminf _{r \rightarrow 0} \frac{\mu(B(x, r))}{r^{\alpha}}>a>0, \quad x \in F .
$$

By Egorov we can find $r_{0}>0$ and a $\mu$-measurable subset $G$ of $F$ such that $\mu(G)>0$ and

$$
\mu(B(x, r))>\frac{a}{2} r^{\alpha}, x \in G \text { and } r \leq r_{0} .
$$

¿From (14) we get, applying (18) twice,

$$
\begin{aligned}
& p_{\alpha}(\mu) \approx \int_{\mathbb{R}^{n}} \int_{\mathbb{R}^{n}} \frac{\mu\left(B\left(x_{3},\left|x_{2}-x_{3}\right|\right)\right)}{\left|x_{2}-x_{3}\right|^{2 \alpha}} d \mu\left(x_{2}\right) d \mu\left(x_{3}\right) \\
& \geq \int_{G} \int_{B\left(x_{3}, r_{0}\right)} \frac{\mu\left(B\left(x_{3},\left|x_{2}-x_{3}\right|\right)\right)}{\left|x_{2}-x_{3}\right|^{2 \alpha}} d \mu\left(x_{2}\right) d \mu\left(x_{3}\right) \\
& \geq \frac{a}{2} \int_{G} \int_{B\left(x_{3}, r_{0}\right)} \frac{d \mu\left(x_{2}\right) d \mu\left(x_{3}\right)}{\left|x_{2}-x_{3}\right|^{\alpha}} \\
& =\frac{a}{2} \int_{G} \int_{0}^{\infty} \mu\left(\left\{x_{2} \in B\left(x_{3}, r_{0}\right):\left|x_{2}-x_{3}\right|^{-\alpha} \geq t\right\}\right) d t d \mu\left(x_{3}\right) \\
& \geq \frac{a \alpha}{2} \int_{G} \int_{0}^{r_{0}} \frac{\mu\left(B\left(x_{3}, r\right)\right)}{r^{1+\alpha}} d r d \mu\left(x_{3}\right) \\
& \geq \frac{a 2 \alpha}{2} \int_{G} \int_{0}^{r_{0}} \frac{d r}{r}=+\infty,
\end{aligned}
$$

which is a contradiction. 
Remark. In Theorem 2.2 of $[\mathrm{M}]$ it is shown that for any finite Borel measure in $\mathbb{C}$, one has the following inequality,

$$
\int_{\mathbb{C}} \int_{\mathbb{C}} \int_{\mathbb{C}} c 2\left(x_{1}, x_{2}, x_{3}\right) d \mu\left(x_{1}\right) d \mu\left(x_{2}\right) d \mu\left(x_{3}\right) \leq C \int_{\mathbb{C}} \int_{0}^{\infty} \frac{\mu(B(x, r)) 2}{r 2} \frac{d r}{r} d \mu(x) .
$$

On the other hand, for $\alpha=1$, there is no general lower inequality like the one in Lemma 2.1. Although we have

$$
c\left(x_{1}, x_{2}, x_{3}\right) \leq \frac{2}{\left|x_{2}-x_{3}\right|}
$$

the reverse inequality may fail very badly. Thus the reverse inequality in (19) does not hold for general measures $\mu$. However, see Theorem 2.3 in [M] where a related result is shown when $\mu$ is the Hausdorff measure associated to some measure function $h$, restricted to some Cantor sets.

We turn now to the proof of the main Theorem.

Proof of the Theorem. We deal first with the inequality

$$
C_{\frac{2}{3}(n-\alpha), \frac{3}{2}}(K) \leq C \gamma_{\alpha_{+}}(K)
$$

Assume that for a probability measure $\mu$ supported on $K$ we have

$$
E_{\frac{2}{3}(n-\alpha), \frac{3}{2}}(\mu)=\int_{\mathbb{R}^{n}} \int_{0}^{\infty}\left(\frac{\mu(B(x, r))}{r^{\alpha}}\right) 2 \frac{d r}{r} d \mu(x) \equiv E<\infty .
$$

Then by Chebyshev, for each $t>0$,

$$
\mu\left\{x \in K: \int_{0}^{\infty}\left(\frac{\mu(B(x, r))}{r^{\alpha}}\right) 2 \frac{d r}{r}>t\right\} \leq \frac{E}{t} .
$$

Taking $t=2 E$, we obtain a compact set $F \subset K$ such that

$$
\int_{0}^{\infty}\left(\frac{\mu(B(x, r))}{r^{\alpha}}\right) 2 \frac{d r}{r} \leq 2 E, \quad x \in F
$$

and

$$
\mu(F) \geq \frac{1}{3} .
$$

If we set $\nu=\mu_{\mid F} / \mu(F)$, then for some positive constant $C$ depending on $\alpha$,

$$
C\left(\frac{\nu(B(x, \rho))}{\rho^{\alpha}}\right) 2 \leq \int_{\rho}^{2 \rho}\left(\frac{\nu(B(x, r))}{r^{\alpha}}\right) 2 \frac{d r}{r} \leq 18 E, \quad x \in F .
$$


To see that $\nu$ satisfies the $\alpha$-growth condition, notice that if $x \notin F$ and $B(x, r) \cap$ $F=\emptyset$, then $\nu(B(x, r))=0$, and if there is some $\xi \in F \cap B(x, r)$, then due to (21)

$$
\nu(B(x, r)) \leq \nu(B(\xi, 2 r)) \leq C r^{\alpha} \sqrt{E} .
$$

Hence we have

$$
M_{\alpha} \nu(x) \leq C \sqrt{E}, x \in \mathbb{R}^{n} .
$$

Then by Lemma 4.2 and Schwarz inequality we get

$$
E_{\alpha}(\nu)=\int_{\mathbb{R}^{n}} U_{\alpha}^{\nu}(x) d \nu(x) \leq C \sqrt{E}+p_{\alpha}(\nu)^{1 / 2} \leq C \sqrt{E} .
$$

Thus, by Lemma 4.1. we obtain

$$
E^{-1 / 2} \leq C E_{\alpha}(\nu)^{-1} \leq C \gamma_{\alpha,+}(K),
$$

which implies (201).

To see the reverse inequality, let $\mu$ be a probability measure supported on $K$ such that

$$
E_{\alpha}(\mu)=\int_{\mathbb{R}^{n}} U_{\alpha}^{\mu}(x) d \mu(x)<\infty .
$$

Since

$$
E_{\alpha}(\mu) \geq \int p_{\alpha}(\mu)(x) d \mu(x),
$$

as before, by Chebyshev,

$$
\mu\left\{x \in K: p_{\alpha}(\mu)(x)>t\right\} \leq \frac{E_{\alpha}(\mu)}{t}, t>0 .
$$

Taking $t=2 E_{\alpha}(\mu)$ we find a compact set $F \subset K$ such that

$$
p_{\alpha}(\mu)(x) \leq 2 E_{\alpha}(\mu), \text { for } x \in F,
$$

and

$$
\mu(F) \geq \frac{1}{3}
$$

Set $\nu=\mu_{\mid F} / \mu(F)$. Then

$$
p_{\alpha}(\nu)=\int_{F} p_{\alpha} 2(\nu)(x) d \nu(x) \leq 36 E_{\alpha}(\mu) 2,
$$

and so, by Lemma 4.2

$$
E_{\alpha}(\mu)^{-1} \leq 6 p_{\alpha}(\nu)^{-1 / 2} \approx E_{\frac{2}{3}(n-\alpha), \frac{3}{2}}(\nu)^{-1 / 2} \leq C_{\frac{2}{3}(n-\alpha), \frac{3}{2}}(K),
$$


which ends the proof of the Theorem.

\section{Acknowledgements}

The authors were partially supported by grants DGI MTM2004-00519 and 2001/SGR/00431 (Generalitat de Catalunya).

\section{References}

[AH] D. R. Adams and L. I. Hedberg, Function Spaces and Potential Theory, Grundlehren der Mathematischen Wissenschaften, 314. Springer-Verlag, Berlin, 1996.

[D] G. David, Analytic capacity, Calderón Zygmund operators, and rectifiability, Publ. Mat, 43 (1999), 3-25.

[G] J. Garnett, Analytic capacity and measure, Lectures Notes in Math. 297. Springer, Berlin-New York, 1972.

[GV] J. Garnett and J. Verdera, Analytic capacity, bilipschitz maps and Cantor sets. Math. Res. Lett. 10 (2003), no. 4, 515-522.

[M] P. Mattila, On the analytic capacity and curvature of some Cantor sets with non- $\sigma$ finite length, Publ. Mat. 40 (1996), 195-204.

[Me] M. S. Melnikov, analytic capacity: discrete approach and curvature of measure, Sbornik: Mathematics 186:6 (1995), 827-846.

[MMV] P. Mattila, M. S. Melnikov and J. Verdera, The Cauchy integral, analytic capacity, and uniform rectifiability, Annals of Mathematics, 144 (1996), 127-136.

[MP] P. Mattila, P. V. Paramonov, On geometric properties of harmonic Lip - $^{-}$ capacity, Pacific J.Math. 171, No. 2 (1995), 469-491.

[MTV] J. Mateu, X. Tolsa and J. Verdera, The planar Cantor sets of zero analytic capacity and the local T(b) theorem, J. Amer. Math. Soc. 16 (2003), no. 1, 19-28.

[MV] M.S. Melnikov and J. Verdera, A geometric proof of the L2 boundedness of the Cauchy integral on Lipschitz graphs, Inter. Math. Res. Not. 7 (1995), 325-331. 
[Pa] H. Pajot, Analytic capacity, Rectifiability, Menger curvature and the Cauchy Integral, Lecture Notes in Math. 1799, Springer, Berlin, 2002.

[Par] P. V. Paramonov, On harmonic approximation in the $\mathcal{C}^{1}$-norm, Math. USSR Sbornik, Vol. 71 (1992), No. 1.

[P] L. Prat, Potential theory of signed Riesz kernels: capacity and Hausdorff measure, to appear in Internat. Math. Res. Notices.

[T1] X. Tolsa, Painleve's problem and the semiadditivity of analytic capacity, Acta Math. 190 (2003), 105-149.

[T2] X. Tolsa, The semiadditivity of continuous analytic capacity and the inner boundary conjecture, to appear in Amer. J. Math.

[T3] X. Tolsa, Bilipschitz maps, analytic capacity and the Cauchy integral, preprint 2003.

[V1] J. Verdera, $\mathcal{C}^{m}$ approximation by solution of elliptic equations, and Calderón-Zygmund operators, Duke Math. J. 55 (1987), 157-187.

[V2] J. Verdera, On the T(1)-theorem for the Cauchy integral, Ark. Mat., 38 (2000), 183-199.

[Vo] A. Volberg, Calderón-Zygmund capacities and operators on nonhomogeneous spaces, CBMS Regional Conference Series in Mathematics. 100. American Mathematical Society, Providence, RI, 2003.

Joan Mateu and Joan Verdera

Departament de Matemàtiques

Universitat Autònoma de Barcelona

08193 Bellaterra (Barcelona)

Spain

E-mail address:mateu@mat.uab.es, jvm@mat.uab.es.

Laura Prat

Departament de Matemàtica Aplicada i Anàlisi, Universitat de Barcelona

Gran Via de les Corts Catalanes, 585

08007 Barcelona

Spain

E-mail address: laura@mat.ub.es 